\title{
NIVELES UMBRALES DE LLUVIA QUE GENERAN DESLIZAMIENTOS: UNA REVISIÓN CRÍTICA
}

\author{
RAINFALL THRESHOLDS FOR THE INITIATION OF LANDSLIDES. \\ A CRITICAL REVIEW
}

\author{
Alfonso Mariano Ramos-Cañón', Mario Germán Trujillo-Vela², Luis Felipe Prada-Sarmiento³
}

Fecha de recepción: 3 de febrero de 2015

Fecha de aprobación: 20 de agosto de 2015

Referencia: A.M. Ramos-Cañón, M.G. Trujillo-Vela, L.F. Prada-Sarmiento. (2015). Niveles umbrales de lluvia que generan deslizamientos: Una revisión crítica. Ciencia e Ingeniería Neogranadina, 25 (2), pp. 61 - 80, D0l: http://dx.doi. org/10.18359/rcin. 1432

\section{RESUMEN}

En el mundo se han propuesto múltiples relaciones lluvia-deslizamientos para sistemas de alerta temprana. Este trabajo presenta una base de datos que sintetiza el estado del arte de umbrales de lluvia que generan movimientos en masa en todo el mundo. Las relaciones más comunes Iluvia-deslizamiento se procesan con el fin de analizar los datos en función de la clasificación climática de Köppen y el área para la cual fue generada. Los resultados muestran la amplia oferta disponible de tipos de parámetros derivados de lluvia que producen deslizamientos, unido con las diferencias considerables en los valores umbrales que pueden tomar dichos parámetros. Estos resultados se condensan en figuras comparativas de niveles umbrales de lluvia que generan deslizamientos propuestos en el mundo para diferentes parámetros. Del análisis de la información recopilada resulta evidente la necesidad de obtener parámetros y umbrales derivados de lluvia que generan deslizamientos para cada zona geográfica y climática específica, de forma tal que se pueda generar una base racional de conocimiento que permita establecer sistemas de alerta temprana de deslizamientos detonados por lluvias con baja variabilidad. Este trabajo aporta en la construcción del conocimiento del catálogo de posibles parámetros derivados de registros de precipitación que permiten generar umbrales de predicción de ocurrencia de deslizamientos. Los investigadores tendrán la posibilidad de buscar relaciones lluvia-deslizamiento, basadas en análisis estadísticos, con una amplia paleta de parámetros, más allá de las variables tradicionales de lluvia antecedente e intensidad ocurrida el día del deslizamiento.

1. I.C., Dr. Ing., Profesor asociado. Pontificia Universidad Javeriana. Bogotá, Colombia, a-ramos@javeriana.edu.co

2. I.A., Estudiante Dr. Ing., Pontificia Universidad Javeriana. Bogotá, Colombia. mario.trujillo@javeriana.edu.co

3. I.C., Dr. Ing., Profesor asistente. Pontificia Universidad Javeriana. Bogotá, Colombia. If.pradas@javeriana.edu.co 
Palabras clave: Relación Lluvia-Deslizamientos, Zonas Climáticas del Mundo, Umbrales de Lluvia.

\begin{abstract}
Multiple rainfall-landslides relationships have been proposed for early warning systems in the world. This work presents a data base that synthesizes the state of the art of rainfall thresholds that generates mass movements in the entire world. The more common rainfall-landslides relationships are processed in order to analyze data in function of the Köppen climatic classification and the area for which it was proposed. The results show a wide range of parameters derived from rainfall that triggers landslides together with considerable differences between threshold values. Data analysis shows the necessity to obtain parameters and thresholds derived from the rainfall that generate landslides for each specific geographical and climatic zone. For that reason, it is possible to generate a rational background that allows us to establish early warning systems of landslides caused by rainfall with low variability. This work provides insights into the catalog of possible parameters derived from rainfall records that allow us to generate thresholds to predict the landslides occurrence. Researchers will have the possibility to search for rainfall-landslide relationships based on statistical analysis, with a wide spectrum of parameters, beyond the traditional variables of antecedent rainfall and the intensity occurred the day of the landslide.
\end{abstract}

Keywords: Rainfall-Landslide Relationship, Climatic zones -Worldwide-, Rainfall Threshold.

\section{INTRODUCCIÓN}

Teniendo presente la necesidad de los centros urbanos para hacer gestión del riesgo de desastres ante amenazas de tipo natural, se hace imprescindible la generación de sistemas de alerta temprana que ayuden a monitorear y anticipar la posible ocurrencia de pérdidas de vidas humanas y materiales debido a la manifestación de la amenaza. Los procesos de remoción en masa generados por lluvia es una de las amenazas de tipo natural que ha generado importantes consecuencias en varios centros poblados. Uno de los aspectos a considerar para el establecimiento de un sistema de alerta temprana es la base de conocimiento del mismo, es decir, es necesario determinar de qué manera se relaciona la precipitación con la generación de deslizamientos en un sitio dado.

Una parte de los procesos de remoción en masa generados por lluvia se presentan debido al incremento en la presión de poros en el talud (o al aumento de la fuerza de infiltración específica) y las condiciones que favorecen o no la generación de deslizamientos se relacionan con las características de los materiales geológicos, historia de la lluvia y geometría del talud, entre otros [1].

La determinación de condiciones de lluvia que detonan deslizamientos se puede hacer con modelos basados en las leyes de la física, los cuales generalmente aplican las leyes de conservación de masa y momentum 
involucrando la infiltración dentro del cuerpo del talud [2]. Los modelos basados en la física requieren la determinación de un número importante de parámetros. Esta labor puede resultar en exceso costosa cuando se quiere aplicar a zonas de gran extensión. Como ventaja, se ha determinado que los modelos físicamente basados presentan un mejor desempeño cuando se aplica en movimientos superficiales en relación con deslizamientos profundos [3].

Esta compleja relación lluvia-deslizamientos también se ha analizado desde la óptica de la experiencia de los evaluadores del riesgo geotécnico, quienes a partir de registros históricos y las características básicas de la lluvia, como la cantidad de precipitación diaria, proponen unos umbrales mínimos a partir de los cuales se pueden desencadenar fenómenos de remoción en masa. En este tipo de aproximaciones, los expertos seleccionan desde su experiencia el tipo de variable derivada de registros de lluvia y generan una explicación basada en la casuística de la relación lluvia-deslizamiento [4-9]. Por otro lado, Caine [10], Glade et al. [11], Jakob y Weatherly [12], Aleotti [13], Guzzetti et al. [3], Guzzetti et al. [14], Brunetti et al. [15], Li et al. [16] y Mathew et al. [17], entre otros, han generado funciones de tipo regresivo $y / 0$ probabilístico para la determinación de lluvias antecedentes, acumuladas, o intensidades de lluvia que generen deslizamientos en aproximaciones dirigidas por datos. En todos los casos mencionados, la incógnita principal radica en determinar cuál es la variable relacionada con lluvia y el nivel umbral que dispara los procesos de remoción en masa. Para poder abordar esta pregunta, es necesario conocer la amplia oferta de parámetros relacionados con lluvia que generan deslizamiento y que se han usado para análisis en diferentes partes del mundo.

En este trabajo se presenta una recopilación de parámetros relacionados con precipitación que han sido usados para establecer los niveles umbrales de lluvia que generan diferentes tipos de deslizamientos. Los umbrales se agrupan en función de la aproximación para la determinación del umbral, el área de cubrimiento y la región climática para la que fue derivada. Se analiza la amplia variabilidad de tipos de umbrales de lluvia y se pone de relieve las ventajas y dificultades que tienen los diferentes tipos de umbrales de lluvia para ser usados como la base de conocimiento de un sistema de alertas tempranas de deslizamientos generados por lluvias.

\section{METODOLOGÍA}

Los diferentes tipos de funciones que relacionan lluvias umbrales y deslizamientos de todo el mundo fueron extraídos de artículos que presentaran una ecuación o un umbral que limita la cantidad de lluvia que se necesita para que se genere un proceso de remoción en masa. La información recopilada se organizó en un listado de 244 funciones que relacionan Iluvia y deslizamientos, reportadas por 60 autores, de donde se extrajo información dividida en cuatro grandes categorías: información bibliográfica, datos geográficos, datos climáticos y tipo de modelo lluvia-deslizamiento.

La categoría de los datos geográficos contiene el nombre de la región o área donde se recopilaron los datos. En los datos climáticos se resume la clasificación climática Köppen basada en precipitación media anual y temperatura media anual, entre otras. 
En los tipos de modelos lluvia-deslizamiento se consigna la información detallada de los umbrales de lluvia que detonan procesos de remoción en masa. En primer lugar, se clasifican los umbrales en cuatro categorías: empíricos, regresivos, probabilísticos y basados en la física. Posteriormente, de la revisión extensiva de las bases de datos referenciales, se encontraron 32 tipos de umbrales que relacionan alguna variable de lluvia con la ocurrencia de deslizamientos. El tipo de umbral se refiere al parámetro o combinación de parámetros derivados de registros de lluvia que se han elaborado para caracterizar la precipitación que genera procesos de remoción en masa. En forma general, estos umbrales se basan en la medición de volúmenes de agua, tiempo o la relación volumen-tiempo. Las definiciones de umbrales de lluvia se consignaron en la tabla 1. Se incluyó, además, la escala espacial a partir de la cual se analizaron los eventos de lluvia para derivar la definición de cada umbral (tabla 2). En este apartado se agruparon los umbrales en cinco categorías de escalas espaciales: global, continental, subcontinental, regional y local.

\section{RESULTADOS}

\subsection{UBICACIÓN GEOGRÁFICA Y RELACIÓN CON CLASIFICACIÓN CLIMÁTICA DE ECUACIONES DE NIVELES UMBRALES DE LLUVIA}

Del análisis de la información recopilada en el estado del arte se obtuvieron 244 ecuaciones, que representan niveles umbrales de parámetros derivados de registros de lluvia que se relacionan con procesos de remoción en masa. La figura 1 presenta la ubicación espacial de los 244 umbrales, representados por los puntos que tienen diferentes colores debido a que fueron graficados en función de las zonas climáticas de Köppen [18]. Con esto se puede ver que los umbrales están generados para zonas montañosas en todo el mundo, y se distribuyen en todos los grupos climáticos principales establecidos por Köppen.

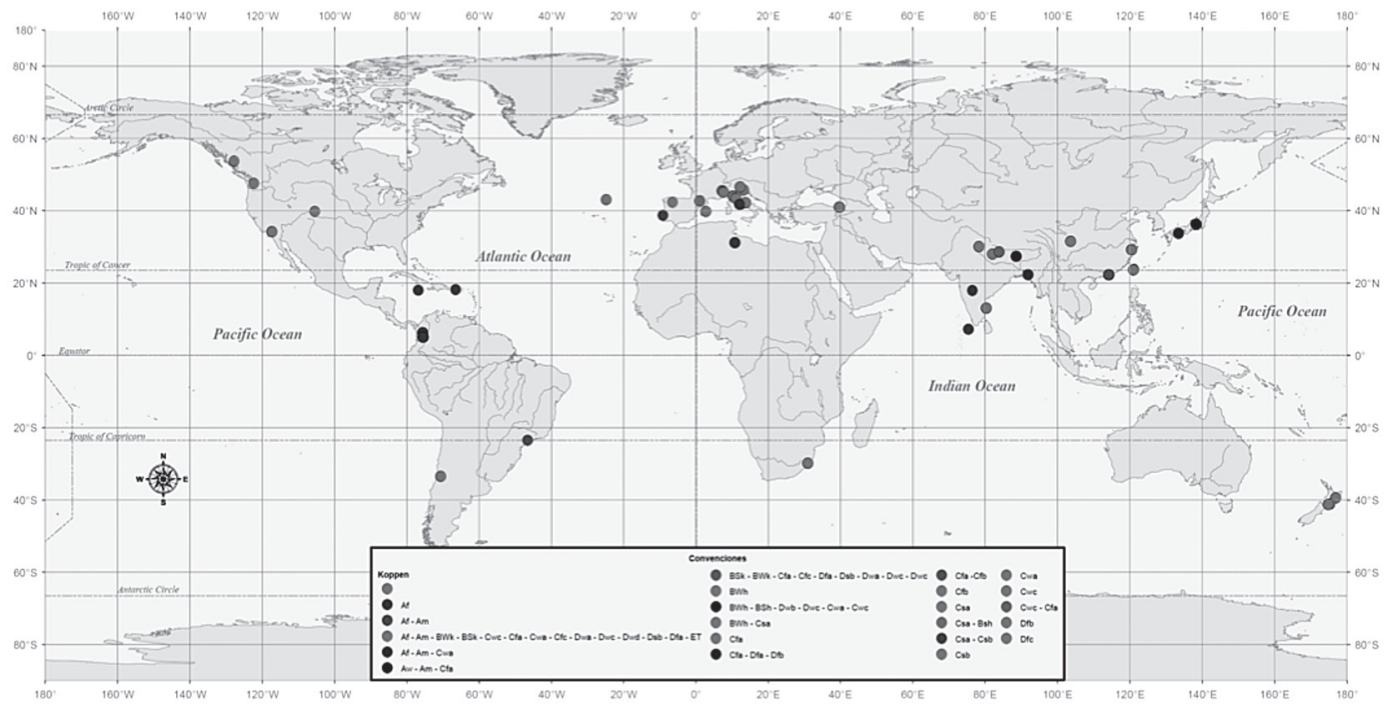

Figura 1. Ubicación y clasificación de los umbrales de lluvia recopilados de la literatura en función de la escala climática de Köppen. 


\subsection{VARIABLES DERIVADAS DE REGISTROS DE PRECIPITACIÓN ASOCIADOS CON DESLIZAMIENTOS}

En la revisión bibliográfica se encontraron 32 tipos de variables derivadas de precipitación que relacionan lluvia-deslizamientos, ya sea en forma de variables independientes, dependientes o por combinación de múltiples variables independientes. El listado se presenta en la tabla 1.

Tabla 1. Definiciones de tipo de variable de precipitación para la iniciación de deslizamientos.

\begin{tabular}{|c|c|c|c|}
\hline Variable & Descripción & Unidades $^{*}$ & Referencia \\
\hline$A_{d}$ & $\begin{array}{l}\text { Lluvia antecedente: Iluvia total acumulada medida } \\
\text { antes del deslizamiento en un periodo determinado en } \\
\operatorname{días~}\left[\mathrm{A}_{d}\right] \text {. }\end{array}$ & {$[\mathrm{mm}]$} & {$[8-9,19-20]$} \\
\hline C & $\begin{array}{c}\text { Lluvia crítica: cantidad total de lluvia desde el momento } \\
\left.\text { de un claro aumento de la intensidad de lluvia [ } \mathrm{t}_{0}\right] \text { para } \\
\text { el momento de la activación del primer deslizamiento } \\
{\left[\mathrm{t}_{\mathrm{f}}\right] \text {. }}\end{array}$ & {$[\mathrm{mm}]$} & {$[21]$} \\
\hline E & $\begin{array}{l}\text { Evento de lluvia acumulada: lluvia total medida desde } \\
\text { el inicio de la precipitación hasta el momento de la falla } \\
\text { [E]. }\end{array}$ & {$[\mathrm{mm}]$} & {$[5,7,19,20,22-29]$} \\
\hline $\mathrm{R}$ & $\begin{array}{l}\text { Lluvia diaria: cantidad total de lluvia para el día del } \\
\text { evento de deslizamiento [R]. }\end{array}$ & {$[\mathrm{mm}]$} & {$[4,6-9]$} \\
\hline$A_{d} D$ & Lluvia antecedente $\left[\mathrm{A}_{\mathrm{d}}\right]$ - duración de la lluvia [D]. & {$[\mathrm{mm}]-[\mathrm{h} \circ \mathrm{d}]$} & {$[30]$} \\
\hline $\mathrm{A}_{\mathrm{d}} \mathrm{N}_{30}$ & $\begin{array}{l}\text { Lluvia antecedente }\left[A_{d}\right] \text { - número de días de lluvia } \\
\text { dentro de los } 30 \text { días en cuestión }\left[N_{30}\right] \text {. }\end{array}$ & {$[\mathrm{mm}]-[\mathrm{d}]$} & [31] \\
\hline$A_{d} P_{1 d}$ & $\begin{array}{c}\text { Lluvia antecedente }\left[\mathrm{A}_{d}\right] \text { : precipitación del día } \\
\text { precedente y cantidad total de lluvia registrada en día } \\
\text { anterior al deslizamiento }\left[\mathrm{P}_{1 d}\right] \text {. }\end{array}$ & {$[\mathrm{mm}]-[\mathrm{mm}]$} & {$[19]$} \\
\hline$A_{d} R$ & Lluvia antecedente $\left[A_{d}\right]$ - Iluvia diaria $[R]$. & {$[\mathrm{mm}]-[\mathrm{mm}]$} & {$[11,32-33]$} \\
\hline$C_{\text {MAP }} \mathrm{D}$ & $\begin{array}{l}\text { Lluvia normalizada critica: división de la lluvia crítica } \\
\text { [C] entre la precipitación media anual [C } C_{\text {MAP }}=\text { C/MAP] - } \\
\text { duración de la lluvia [D]. }\end{array}$ & {$[-]-[h \circ d]$} & {$[13,21]$} \\
\hline DNSR & $\begin{array}{l}\text { Duración de la lluvia [D] - precipitación de tormenta } \\
\text { normalizada. Cantidad de eventos de lluvia dividida } \\
\text { entre la precipitación media anual [NSR=NS/MAP]. }\end{array}$ & {$[\mathrm{h}]-[1 / \mathrm{mm}]$} & [34] \\
\hline$E A_{30 d}$ & $\begin{array}{l}\text { Evento de lluvia acumulativa [E] - Iluvia antecedente de } \\
30 \text { días }\left[\mathrm{A}_{30 \mathrm{~d}}\right] \text {. }\end{array}$ & {$[\mathrm{mm}]-[\mathrm{mm}]$} & {$[31]$} \\
\hline ED & Evento de lluvia acumulativa [E] - duración de lluvia [D]. & {$[\mathrm{mm}]-[\mathrm{h} \circ \mathrm{d}]$} & {$[10,24,29,35,36]$} \\
\hline
\end{tabular}




\begin{tabular}{|c|c|c|c|}
\hline$E_{\text {MAP }}$ & $\begin{array}{l}\text { Evento de lluvia acumulativa normalizada. Evento de } \\
\text { lluvia acumulada dividida entre la precipitación media } \\
\left.\text { anual [ } \mathrm{E}_{\mathrm{MAP}}=\mathrm{E} / \mathrm{MAP}\right] \text {. }\end{array}$ & {$[-]$} & {$[19,37]$} \\
\hline$E_{\text {MAP }} D$ & $\begin{array}{c}\text { Evento de lluvia acumulativa normalizada [E } \\
\text { duración de la lluvia [D]. }\end{array}$ & {$[-]-[h \circ d]$} & {$[34,38]$} \\
\hline$E_{M A P} \mid$ & $\begin{array}{l}\text { Evento de lluvia acumulativa normalizada [E } \\
\text { intensidad de la lluvia [I]. }\end{array}$ & {$[-]-[\mathrm{mm} / \mathrm{h}]$} & {$[34,39]$} \\
\hline$E P_{1 d}$ & $\begin{array}{c}\text { Evento de lluvia acumulativa [E] - precipitación del día } \\
\text { precedente. Cantidad total de lluvia registrada en día } \\
\text { anterior al deslizamiento }\left[\mathrm{P}_{1]}\right] \text {. }\end{array}$ & {$[\mathrm{mm}]-[\mathrm{mm}]$} & [19] \\
\hline $\mathrm{ID}$ & $\begin{array}{c}\text { Intensidad de lluvia: división de la intensidad de lluvia } \\
\text { [I] entre la precipitación media anual [I } \\
\text { duración de la lluvia [D]. }\end{array}$ & {$[\mathrm{mm} / \mathrm{h}]-[\mathrm{h} \circ \mathrm{d}]$} & $\begin{array}{l}{[3,10,12,14,15} \\
17,23,31,34,38 \\
40-60]\end{array}$ \\
\hline IE & $\begin{array}{c}\text { Intensidad de lluvia [I] - evento de lluvia acumulativa } \\
\text { [E]. }\end{array}$ & {$[\mathrm{mm} / \mathrm{h}]-[\mathrm{mm}]$} & {$[61,62]$} \\
\hline$I_{\text {MAP }}-C_{\text {MAP }}$ & $\begin{array}{l}\text { Intensidad de Iluvia normalizada }\left[\mathrm{I}_{\text {MAP }}\right] \text { - Iluvia critica } \\
\text { normalizada }\left[\mathrm{C}_{\text {MAP }}\right]\end{array}$ & {$[1 / h]-[-]$} & [13] \\
\hline$I_{\text {MAP }} D$ & $\begin{array}{l}\text { Intensidad de lluvia normalizada: división de la } \\
\text { intensidad de lluvia entre la precipitación media anual } \\
{\left[I_{\text {MAP }}=1 / \text { MAP] - duración de la lluvia [D]. }\right.}\end{array}$ & {$[1 / h]-[h \circ d]$} & $\begin{array}{c}{[3,13,14,38-39,47} \\
57,60]\end{array}$ \\
\hline INSR & $\begin{array}{l}\text { Intensidad de la lluvia [I] - precipitación de tormenta } \\
\text { normalizada. Cantidad de eventos de lluvia dividida } \\
\text { entre la precipitación media anual [NSR=NS/MAP]. }\end{array}$ & {$[\mathrm{mm} / \mathrm{h}]-[1 / \mathrm{mm}]$} & {$[34]$} \\
\hline RDN & $\begin{array}{c}\text { Días lluviosos normales: relación entre la precipitación } \\
\text { media anual y el número medio de días de lluvia en un } \\
\left.\text { año [RDN=MAP/N } N_{365}\right] \text {. }\end{array}$ & {$[\mathrm{mm} / \mathrm{h}]$} & {$[3,14,17]$} \\
\hline IRDND & $\begin{array}{l}\text { Intensidad de lluvia normalizada: división de la } \\
\text { intensidad de lluvia [I] entre el número de días Iluviosos } \\
\text { normales [IRDND=I/RDN]- duración de la lluvia [D]. }\end{array}$ & {$[\mathrm{mm} / \mathrm{h}]-[\mathrm{h} \circ \mathrm{d}]$} & {$[3,14,17]$} \\
\hline$L_{C F} E R$ & $\begin{array}{l}\text { Frecuencia acumulada de ocurrencia de deslizamientos: } \\
\text { representa la probabilidad acumulada de distintos } \\
\text { niveles de actividad de deslizamientos durante un } \\
\text { evento de lluvia } R\left[L_{C F} \text { - Evento de lluvia acumulativa }\right. \\
{[\text { [E] - Lluvia Diaria [R]. }}\end{array}$ & {$[\%]-[\mathrm{mm}]-[\mathrm{mm}]$} & {$[16]$} \\
\hline$L_{C F} R$ & $\begin{array}{l}\text { Frecuencia acumulada de ocurrencia de deslizamientos. } \\
\qquad\left[\mathrm{L}_{\mathrm{CF}}\right] \text { - Iluvia diaria }[\mathrm{R}] .\end{array}$ & {$[\%]-[\mathrm{mm}]$} & {$[16]$} \\
\hline$M A_{\triangleright} E A_{d}$ & $\begin{array}{l}\text { Valor de discriminación para grandes eventos: una } \\
\text { función discriminante para clasificar los eventos de Iluvia } \\
\text { que activan } 10 \text { deslizamientos o más }\left[M A_{\triangleright}\right] \text { - evento de } \\
\text { Iluvia acumulativa }[E] \text { - Iluvia antecedente }\left[A_{d}\right] \text {. }\end{array}$ & & [63] \\
\hline
\end{tabular}




\begin{tabular}{|c|c|c|c|}
\hline $\mathrm{Ml}_{D} E \mathrm{~A}_{d}$ & $\begin{array}{l}\text { Valor de discriminación de evento menor: una función } \\
\text { discriminante para clasificar los eventos de lluvia que } \\
\text { activan menos de } 10 \text { deslizamientos }\left[\mathrm{MI} \mathrm{D}_{\mathrm{D}} \text { - evento de }\right. \\
\quad \text { Iluvia acumulativa [E] - Iluvia antecedente }\left[\mathrm{A}_{d}\right] \text {. }\end{array}$ & & [63] \\
\hline$L_{N} E$ & $\begin{array}{l}\text { Número de deslizamientos }\left[L_{N}\right] \text { - evento de lluvia } \\
\text { acumulativa }[E] \text {. }\end{array}$ & {$[-]-[\mathrm{mm}]$} & [63] \\
\hline$L_{p} R A_{d}$ & $\begin{array}{c}\text { Probabilidad de ocurrencia de deslizamiento: } \\
\text { probabilidad de ocurrencia de deslizamientos dada } \\
\text { todas las combinaciones posibles de lluvia diaria }\left[\mathrm{L}_{p}\right] \text { - } \\
\text { Iluvia diaria }[R] \text { - Iluvia antecedente }\left[\mathrm{A}_{d}\right] \text {. }\end{array}$ & {$[\%]$} & {$[11]$} \\
\hline$F_{p} R E A_{d}$ & $\begin{array}{c}\text { Probabilidad de ocurrencia de falla: probabilidad de } \\
\text { falla de un talud en función de un conjunto variables } \\
\text { independientes conservadas en el modelo de regresión } \\
\text { logística }\left[\mathrm{F}_{\mathrm{p}} \text { - Lluvia diaria [R] - evento de lluvia }\right. \\
\text { acumulativa [E]- Iluvia antecedente }\left[\mathrm{A}_{d}\right] \text {. }\end{array}$ & & {$[17]$} \\
\hline $\mathrm{R}_{0} \mathrm{~A}_{6}$ & $\begin{array}{l}\text { Precipitación intradía: precipitación total del día en que } \\
\text { se produjo el deslizamiento }\left[\mathrm{R}_{0}\right] \text { - precipitación efectiva } \\
\text { antecedente de } 6 \text { días antes del deslizamiento }\left[\mathrm{A}_{6 \mathrm{~d}}\right] \text {. }\end{array}$ & {$[\mathrm{mm}]-[\mathrm{mm}]$} & {$[64]$} \\
\hline $\mathrm{RA}_{\mathrm{d}} \mathrm{RM}_{\mathrm{nO}}$ & $\begin{array}{l}\text { Lluvia diaria }[\mathrm{R}] \text { - Iluvia antecedente }\left[\mathrm{A}_{\mathrm{d}}\right] \text { - precipitación } \\
\text { regional máxima el día nantes del día de deslizamiento } \\
\qquad\left[\mathrm{RM}_{\mathrm{no}}\right] \text {. }\end{array}$ & & {$[6]$} \\
\hline $\mathrm{RA}_{25} \mathrm{SL}$ & $\begin{array}{l}\text { Lluvia diaria }[R] \text { - Iluvia antecedente de } 25 \text { días del } \\
\text { deslizamiento }\left[\mathrm{A}_{25}\right] \text { - nivel de nevadas. Cantidad de } \\
\text { precipitación de pequeños cristales de hielo [SL] }\end{array}$ & {$[\mathrm{mm}]-[\mathrm{mm}]-[\mathrm{mm}]$} & [65] \\
\hline $\mathrm{Rl}_{\mathrm{R}} \mathrm{E}$ & $\begin{array}{c}\text { Indice numérico de riesgo por precipitación. Mide la } \\
\text { posibilidad de ocurrencia de deslizamientos }\left[\left.R\right|_{R}\right] \text { - } \\
\text { Evento de lluvia acumulativa }[E]\end{array}$ & {$[\mathrm{mm}]-[\mathrm{mm}]$} & {$[28]$} \\
\hline
\end{tabular}

*h=horas, d=días, mm=milímetro

\subsection{ESCALA ESPACIAL DE CUBRIMIENTO DE LAS ECUACIONES QUE RELACIONAN LLUVIA Y DESLIZAMIENTO}

Los umbrales pueden ser clasificados por extensión (área), para la cual fueron generados en local, regional, subcontinental, continental y global (tabla 2). Los umbrales para pequeñas escalas, como la local, son considerados para una configuración climática y geomorfológica local. Los umbrales regionales, para zonas con características meteorológicas, climáticas y fisiográficas similares. Los umbrales para escala subcontinental, continental y global establecen un mínimo nivel de precipitación para grandes extensiones o general (para todo el planeta), por debajo del cual no se producen deslizamientos [3].

Las escalas subcontinental, continental y global pueden ser relevantes y aplicables de forma indicativa cuando no se han generado estudios a escala espacial, regional o local. Sin embargo, el uso de escalas mayores a la regional en una zona específica puede generar numerosas falsas alarmas. Es decir, la aplicabilidad de los 
Tabla 2. Clasificación de escala de umbrales según el área de estudio.

\begin{tabular}{|c|c|}
\hline Escala & Área \\
\hline Local & $<1.000 \mathrm{~km}^{2}$ \\
\hline Regional & $1.000 \mathrm{~km}^{2}<\mathrm{a}<100.000 \mathrm{~km}^{2}$ \\
\hline Subcontinental & $100.000 \mathrm{~km}^{2}<\mathrm{a}<1^{\prime} 000.000 \mathrm{~km}^{2}$ \\
\hline Continental & $1^{\prime} 000.000 \mathrm{~km}^{2}<\mathrm{a}<10^{\prime} 000.000 \mathrm{~km}^{2}$ \\
\hline Global & $>10^{\prime} 000.000 \mathrm{~km}^{2}$ \\
\hline
\end{tabular}

umbrales generados a escala subcontinental a global tiene un carácter limitado para que sean usados como base de conocimiento para sistemas de alertas tempranas de deslizamientos disparados por lluvias.

\subsection{FRECUENCIA DE TIPOS DE UMBRALES DE LLUVIA QUE GENERAN DESLIZAMIENTO}

La figura 2 muestra que uno de los tipos de umbrales de lluvia que se reporta con mayor frecuencia en la literatura es la relación intensidad-duración (ID) con un 35,2 \% de la base de datos, lo que representa 86 ecuaciones de las 244 ecuaciones recopiladas en total. Este tipo de umbral es ampliamente usado dado que genera una relación sencilla de análisis, que además también ha sido usada ampliamente en hidrología para determinar intensidades de tormenta.

En segundo lugar se encuentra la lluvia acumulada para varios días (E). La lluvia acumulada es un parámetro conveniente cuando los pluviómetros no tienen definición a escala horaria ni sub-horaria como para utilizar el parámetro de intensidad-duración. El número de días de lluvia acumulada varía fuertemente entre las diferentes propuestas.

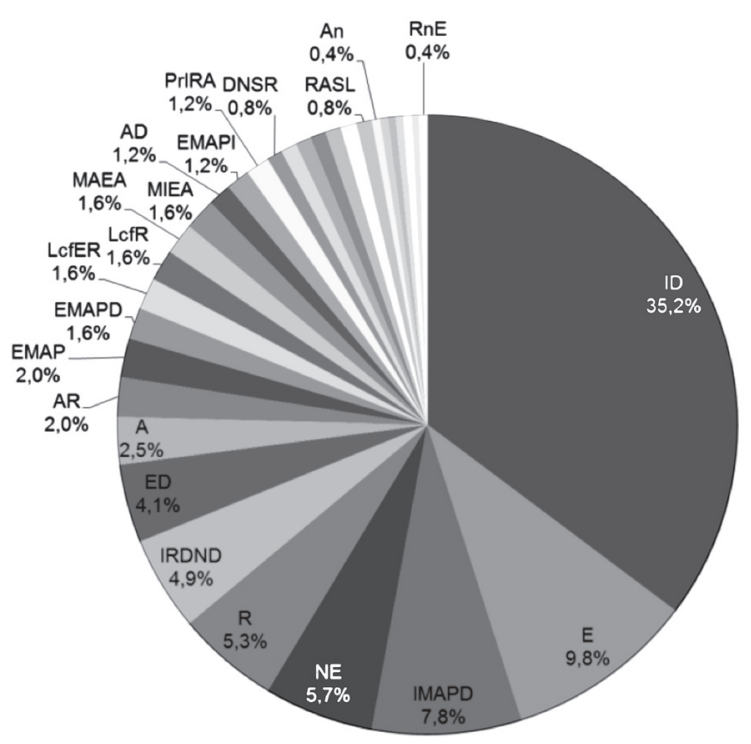

Figura 2. Estadísticas de la sistematización de la base de datos de los artículos por tipos umbrales de lluvia.

En la figura 3a se presenta la clasificación de los umbrales según la escala espacial de aplicación. Se puede apreciar que la mayor parte de los estudios, dependiendo de la escala espacial, son de tipo local, con 154 ecuaciones que representan un $63 \%$ de la base de datos. Se ha visto que esta escala es la más usada cuando el objetivo del planteamiento del nivel umbral de lluvia es la de ser la base de conocimiento para un sistema de alerta temprana [3], ya 


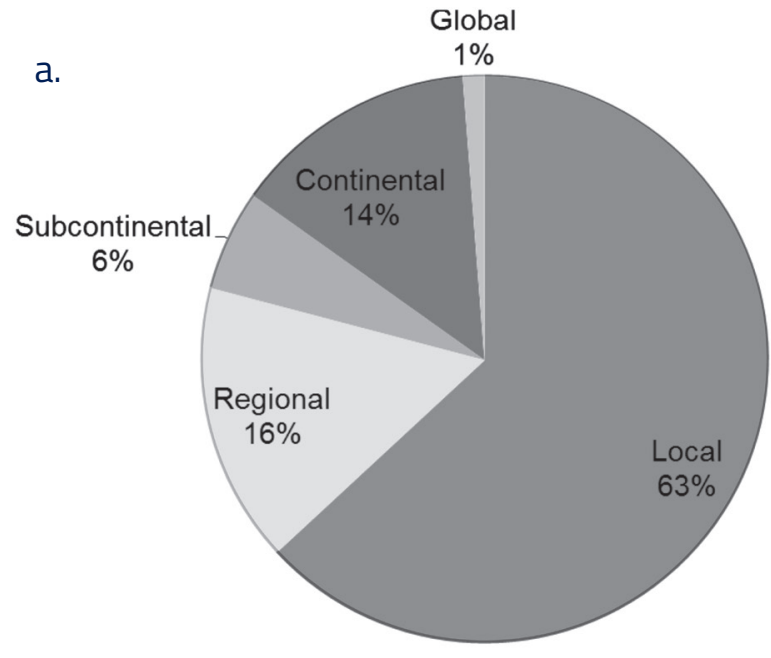

Figura 3a. Escala espacial de aplicabilidad de umbrales.

que con este nivel de cobertura espacial, el nivel umbral es definido de forma que tenga en cuenta intrínsecamente las condiciones geológicas, geomorfológicas y geotécnicas de la zona. Adicionalmente, se espera que el régimen pluviométrico en esta escala espacial de cobertura no presente altas variabilidades, y que estas no se vean regularizadas al emplear valores promedio obtenidos de análisis realizados en escalas espaciales más grandes.

En la figura 3b se presenta el tipo de modelo con el que se propuso el nivel umbral, donde se evidencia que uno de los tipos de modelo más utilizado para la generación o propuesta de un nivel de umbral de lluvia relacionada con un proceso de remoción en masa es del tipo regresivo, con 159 ecuaciones, lo cual representa un 59,3\% de toda la base de datos. Le sigue en orden de importancia el tipo empírico, con el $20 \%$ de los datos, y en tercer lugar se encuentra el de tipo estocástico. Se recalca el hecho que estos tres tipos de modelo utilizan el mismo tipo de información histórica base y la diferencia entre estos radica en el nivel

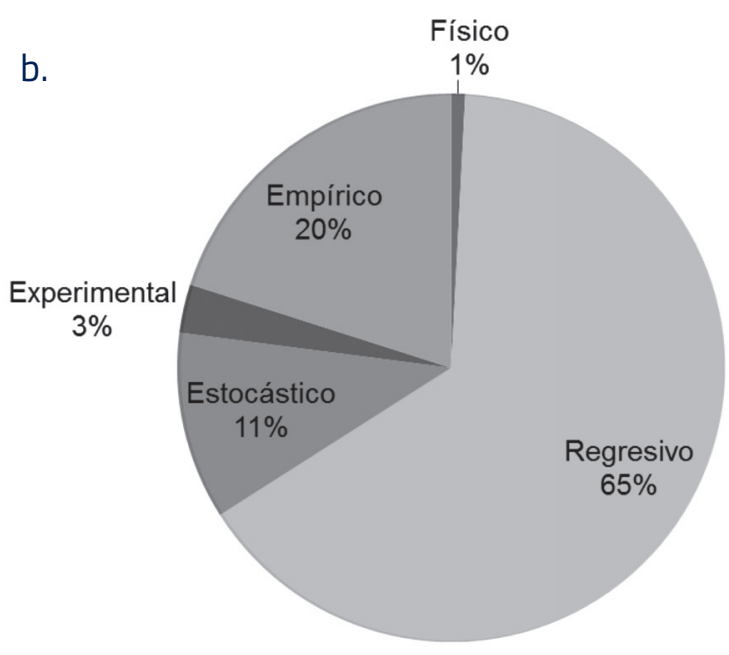

Figura 3b. Tipo de modelo del cual se basan los umbrales

de complejidad del análisis matemático de los datos que va en orden ascendente partiendo de los modelos empíricos simples, pasando por los modelos regresivos y terminando con los modelos más sofisticados de tipo estocástico.

\subsection{VARIACIÓN DE LAS ECUACIONES QUE RELACIONAN NIVELES UMBRALES DE LLUVIA PARA DIFERENTES PARTES DEL MUNDO}

Los umbrales más usados por los investigadores en el mundo, como ID, E, I MAP $_{\text {, }} \mathrm{D}$ $R, E D$, que contabilizan el $68 \%$ de los umbrales recopilados en este trabajo, fueron graficados de la figura 4 a la 8, haciendo una discriminación en términos de la escala climática de Köppen y escala espacial de aplicación.

El límite inferior de todas las ecuaciones propuestas en términos de la relación intensidad-duración ID (figura 4) se presenta como un nivel base en el que se prevé que un evento de lluvia por debajo de este valor umbral no tendrá la capacidad de generar un 

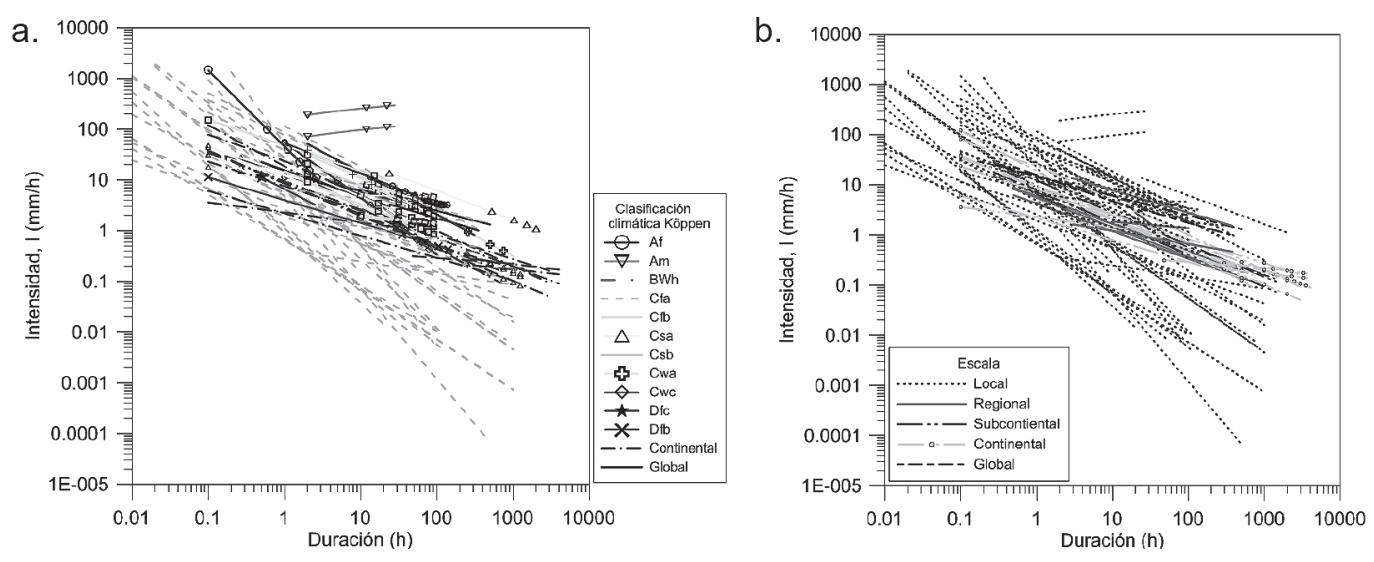

Figura 4. Relación intensidad-duración (ID) en función de a. clasificación climática de Köppen; b. escala de aplicación espacial.

proceso de remoción en masa, independiente del tipo de material, geología o geomorfología de la zona. Por otro lado, se observa que el límite inferior de las curvas ID generadas para distintos ambientes climáticos dado por la clasificación climática de Köppen está modulado por los climas templados subtropicales sin estación seca (Cfa) y clima oceánico (Cfb). La clasificación climática para la sabana de Bogotá es Cfb, por lo que los niveles umbrales mínimos reportados en la figura 4 pueden servir como referente en términos de comparación. Aunque se graficaron también unos umbrales ID para escalas subcontinental, continental y global (figura 4b) se puede apreciar cómo estos umbrales generados para escalas espaciales tan grandes, no produjeron un mínimo por debajo del cual no se producen deslizamientos, ya que por debajo de estos umbrales de gran escala (líneas cafés, amarillas y negras, respectivamente) sí se presentan umbrales inferiores en los cuales se pueden presentar eventos de movimientos de ladera. Las diferencias pueden ser de órdenes de magnitud. Esto indicaría que de querer producir un mínimo para grandes escalas (subcontinental, continental y global), se requiere de la mayor cantidad de umbrales clasificados por tipo (ej. ID), para así seleccionar los puntos más bajos del conjunto de umbrales, y generar la ecuación que representa la curva del conjunto de puntos mínimos, pero nuevamente esto podría generar falsas alarmas de deslizamientos (falsos positivos).

El tipo de umbral graficado en la figura 5 relaciona la lluvia acumulada antes de la ocurrencia del proceso de remoción en masa en un tiempo dado (abscisas). La mayoría de autores que usan esta definición de umbral lo plantean como modelos empíricos dado que usualmente el análisis lo realizan para eventos discretos de deslizamientos disparados por lluvia. Se observa que para la totalidad de datos analizados en el estado del arte, la lluvia mínima acumulada para que se presenten procesos de remoción en masa es de $15 \mathrm{~mm}$, con duraciones entre 20 y 100 horas. Por otro lado, la mayoría de umbrales de este tipo (lluvia acumulada) son desarrollados para la escala espacial local. Una de las ventajas que tiene el uso de este parámetro es su fácil 

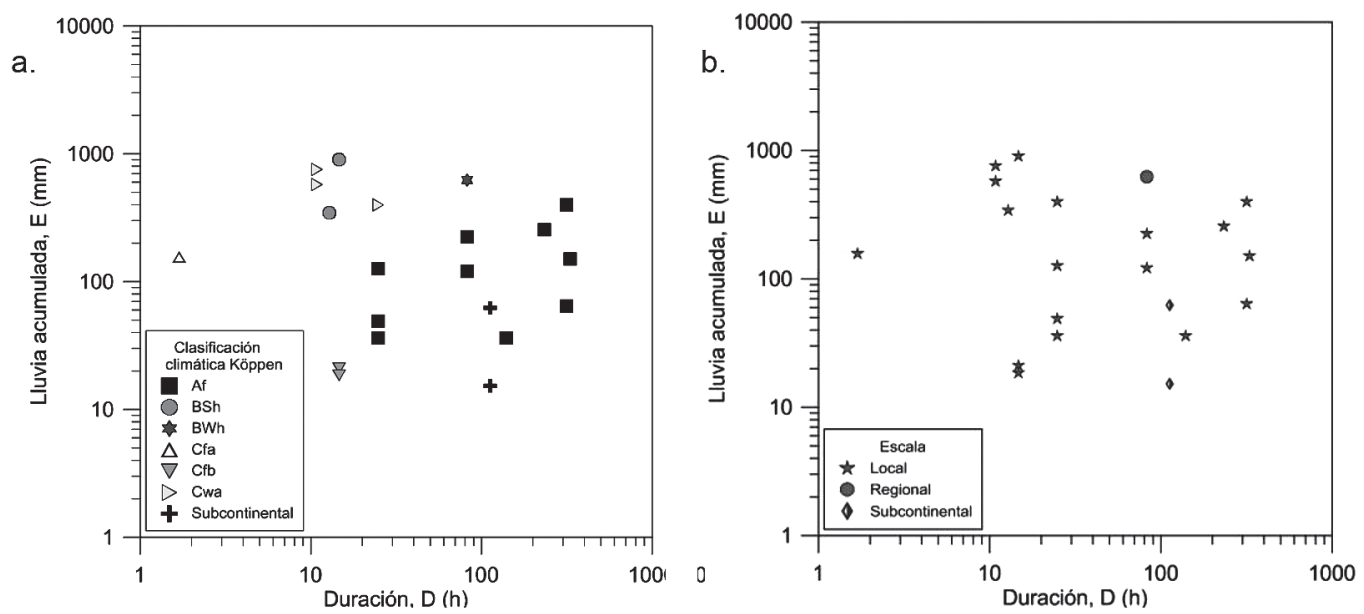

Figura 5. Umbral Iluvia acumulada (E) con la duración asignada en cada caso en función de a. clasificación climática de Köppen; b. escala de aplicación espacial.

determinación con ayuda de instrumentación pluviométrica convencional a nivel horario y superior (diaria).

Los umbrales obtenidos con la relación ED se pueden representar con ecuaciones lineales con pendientes positivas al ajustar la distribución de puntos obtenida en esta recopilación bibliográfica.



En la figura 6a (intensidad normalizadaduración $I_{M A P} D$ ), no se evidencia una tendencia clara de agrupamiento de curvas en función de la clasificación climática. La figura $6 \mathrm{~b}, \mathrm{a}$ diferencia de la figura $5 b$, el umbral $I_{\text {MAP }} D$ distingue que los valores más bajos hacen referencia a escalas espaciales mayores (continental y regional), en tanto que los umbrales determinados para condiciones de

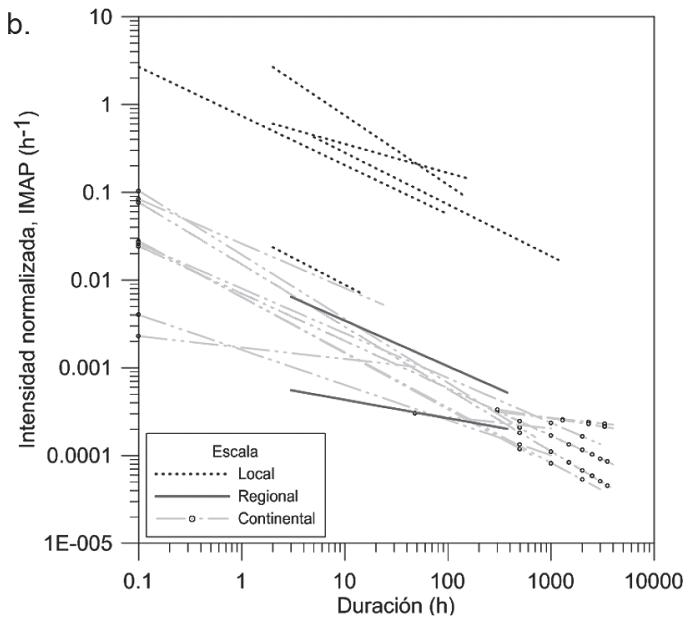

Figura 6. Relación intensidad normalizada-duración ( $\left.l_{\text {MAP }} D\right)$ en función de a. tipo de modelo; b. escala de aplicación espacial c. clasificación climática de Köppen. 
escala espacial local son mucho mayores. En la mayoría de casos, los niveles umbrales $I_{\text {MAP }} D$ locales son mayores en un orden de magnitud para una duración dada con relación a los generados para escala continental o aún regional.

En la figura 7 se puede observar que el tipo de umbral en función únicamente de la lluvia diaria acumulada ha sido usado cuando la escala de análisis espacial es local. Un valor mínimo de 10 mm determina el umbral para la totalidad de la base de datos. Los autores que han usado este tipo de umbral lo han aplicado a zonas climáticas semejantes a la de Bogotá (Cfb). Sin embargo, también se nota la alta variabilidad del nivel umbral de lluvia diaria (R) para una misma zona climática (Cfb), ya que puede tener diferencias de un orden de magnitud.

El tipo de umbral ED (Figura 5) tiene semejanza con el umbral tipo E mostrado en la figura 5.
En ambos umbrales (figuras 5 y 8) se utilizan las variables lluvia acumulada y duración. Una diferencia radica en que los umbrales propuestos en la figura 5 son de carácter discreto, mientras que los propuestos en la figura 8 se tratan de una propuesta continua, válida para un amplio rango de duraciones. La otra diferencia se centra en que las curvas propuestas en la figura 8 delimitan zonas en donde se espera que ocurra deslizamiento una vez ha pasado el umbral. El umbral definido en términos de cobertura espacial de tipo global abarca rangos de duración que comprenden al menos tres órdenes de magnitud, tanto en duración (0.1 h a 1.000 h) como en Iluvia acumulada (10 mm a 1.000 mm). Por otro lado, queda explícito en las relaciones mostradas en la figura 8 que hay una relación de proporcionalidad directa entre el tiempo de duración de la lluvia antecedente y la cantidad de lluvia para que se genere deslizamiento. Situación que no se explicita en la propuesta discreta de la figura 5. a.



b.

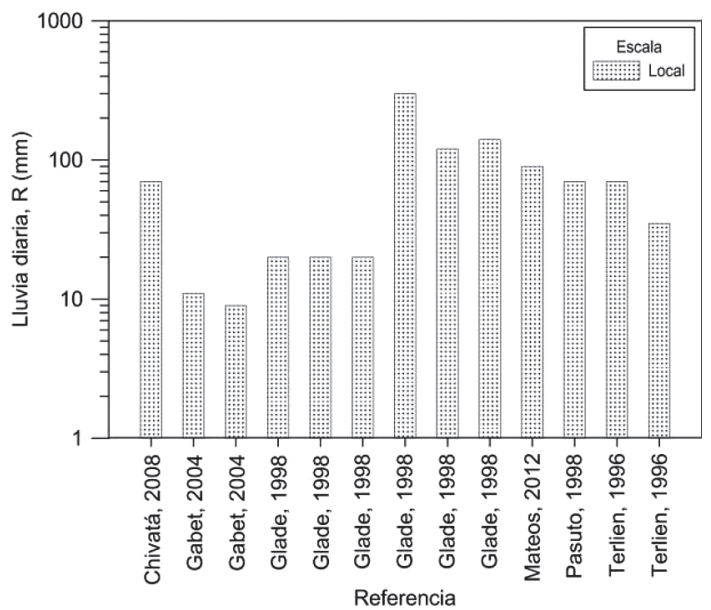

Figura 7. Umbral Iluvia diaria (R) en función de a. clasificación climática de Köppen; b. escala de aplicación espacial. 

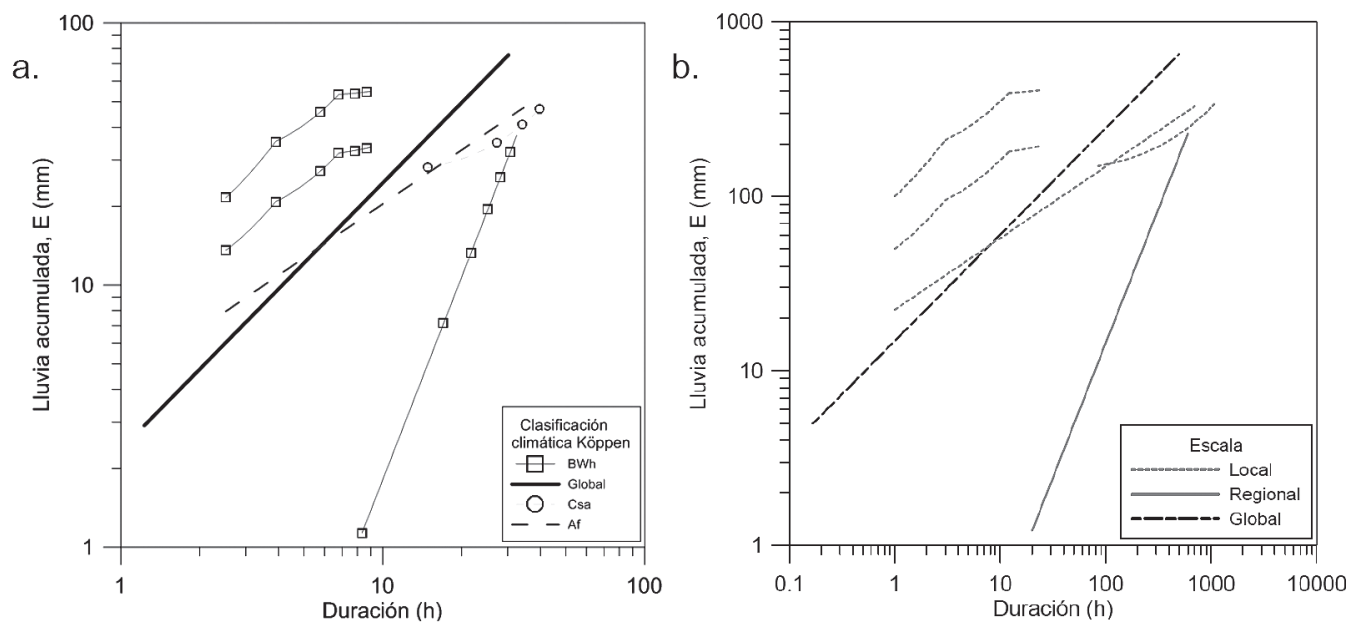

Figura 8. Relación Lluvia acumulada-duración (ED) en función de a. clasificación climática de Köppen; b. escala de aplicación espacial.

\section{DISCUSIÓN}

Los umbrales deben ser generados en mejor medida para escalas locales de modo que tenga en cuenta intrínsecamente las condiciones geológicas, geomorfológicas y geotécnicas de la zona. Además, es importante tener un registro histórico de eventos de lluvia que hayan generado deslizamientos lo suficientemente grandes para generar relaciones de tipo estocástico, donde a partir de las variables de entrada se pueda determinar una probabilidad de generación de deslizamientos dado uno o un conjunto de variables relacionadas con lluvia.

Tanto en la información mostrada en la figura 5 como en la figura 8 se observa que la duración de la lluvia antecedente varía desde una hora hasta 20 días. La variabilidad de los días antecedentes es alta, y es una pregunta que debe responder el modelador si selecciona la variable lluvia antecedente como aquella que representa la relación lluvia deslizamiento. Son dos variables en la relación lluvia deslizamiento en función de la lluvia antecedente: es decir, el deslizamiento en una zona dada se dispara cuando la lluvia acumulada de $X$ días sobrepasa $Y$ volumen.

Para determinar los umbrales de gran escala (subcontinental, continental o global) se debe obtener la mayor cantidad posible de umbrales de cada tipo, para poder seleccionar los puntos más bajos de las relaciones y generar la ecuación que mejor se ajuste a dichos puntos más bajos del conjunto. Sin embargo, como se ha venido insistiendo en el desarrollo del trabajo, estas relaciones generadas para grandes extensiones de tierra pueden perder el contenido intrínseco de las condiciones geológicas, geomorfológicas y geotécnicas de la zona, produciendo así múltiples falsas alarmas por deslizamientos. Con esto se hace necesario generar umbrales de lluvia diferenciados para procesos de remoción en masa de tipo superficial (flujos de detritos, flujos de lodos), deslizamientos profundos y otros relacionados con caída de rocas y bloques. Adicionalmente, se espera que los 
niveles umbrales generados a nivel global sean conservadores con relación a los generados a nivel local. Es decir, generan falsos positivos si se aplican las relaciones globales a una zona de menos de $1.000 \mathrm{~km}^{2}$.

García et al. [66] plantean que, al proponer niveles umbrales de lluvia a través de la relación de dos variables, estas deben ser estadísticamente independientes entre sí para mejorar los resultados predictivos de un modelo estocástico, pero dos o más parámetros cualquiera derivados de la lluvia en una misma zona geográfica deben presentar cierto grado de correlación por lo que se espera que no sean variables independientes. En este caso es importante determinar el grado de correlación permisible o "independencia" entre dichas variables que mejoran la predictibilidad de un modelo de tipo estocástico, a diferencia de las que estén mejor correlacionadas.

Diferentes autores proponen que, dependiendo del tipo de proceso de remoción en masa característico de una zona, se debe hacer la selección de las variables relacionadas con Iluvia para su análisis. Para ejemplo de esto, la relación intensidad-duración se toma típicamente cuando se hace análisis de flujos de detritos o de material desagregado. Caine [10] presenta una interesante recopilación bibliográfica de 73 puntos que relacionan intensidad y duración donde se generaron flujos de detritos de artículos publicados desde 1943 a 1979 en Europa. La generación de movimientos en masa con fallas profundas podría estar mejor relacionada con variables de lluvia que impliquen largos tiempos, como la lluvia antecedente de varios días.

Es importante tener presente que la aproximación de umbrales de lluvia que disparan deslizamientos implica el supuesto de una discontinuidad temporal en la lluvia. En la mayoría de los casos analizados en este trabajo se define que no se generan procesos de remoción en masa si el o los parámetros relacionados con lluvia están por debajo del nivel umbral definido. Una vez la precipitación es tal que supera el umbral, se dice que se presenta el proceso de remoción en masa. Esta aproximación presenta dos deficiencias:

- La primera de estas es que la definición anteriormente usada supone que la generación del proceso de remoción en masa es un proceso discontinuo, es decir, que el deterioro de la resistencia al corte de los materiales no es gradual sino que su resistencia cae de forma abrupta, o que el incremento del peso de la masa potencialmente deslizada debido a la Iluvia varía en forma intempestiva. Esta suposición se aleja de las observaciones, dado que las variables relacionadas con la estabilidad de taludes varían de forma continua y derivable.

- La segunda deficiencia que se observa con la definición general del nivel umbral de Iluvia, al suponer que tanto los parámetros geomecánicos como los de precipitación presentan una variabilidad espaciotemporal intrínseca, debido a que estos procesos varían en el espacio y en el tiempo. Así, cualquier modelo que relaciona lluvia y procesos de remoción en masa debería tener presente la cuantificación de la incertidumbre espacio-temporal de estos dos procesos.

Dadas las dos deficiencias anteriormente mencionadas, se considera que la propuesta de nivel umbral de lluvia que dispara procesos 
de remoción en masa debe estar presentada en términos probabilísticos o posibilísticos en lugar de estar en términos de un umbral de tipo determinístico.

A pesar de que se establece que las relaciones Iluvia deslizamiento determinadas a nivel local capturan las condiciones geológicas, geomorfológicas, geotécnicas e hidrológicas, es necesario mencionar que en zonas geográficas de montaña en el trópico, como el área urbana de Bogotá con áreas de menos de $1.000 \mathrm{~km}^{2}$, el régimen pluviométrico varía considerablemente y las condiciones geológicas también. Dado lo anterior, la generación de la relación Iluvia deslizamiento para Bogotá debe hacerse con áreas menores a $1.000 \mathrm{~km}^{2}$ para que pueda ser representativa del tipo de movimiento y sea utilizable para la generación de un sistema de alerta temprana.

\section{AGRADECIMIENTOS}

Los autores agradecen a la Pontificia Universidad Javeriana por el apoyo bajo el proyecto de investigación No. 6143 "Determinación de niveles umbrales de Iluvia que generan deslizamientos en Bogotá". La parte inicial del presente trabajo se enmarcó en el convenio de cooperación entre el Instituto Geofísico Universidad Javeriana y el Idiger (Instituto Distrital de Gestión de Riesgos y Cambio Climático).

\section{BIBLIOGRAFÍA}

[1] Wieczorek, G. F. (1996). Landslide triggering mechanisms. Landslides: Investigation and Mitigation, pp. 76-90.
[2] Iverson, R. M. (2000). Landslide triggering by rain infiltration. Water Resources Research, 36(7):1897-1910.

[3] Guzzetti, F., Peruccacci, S., Rossi, M. and Stark, C. P. (2007). Rainfall thresholds for the initiation of landslides in central and southern Europe. Meteorology and Atmospheric Physics, 98(3-4):239-267. DOI: 10.1007/s00703-007-0262-7.

[4] Chivatá Cárdenas, I. (2008). Using multinomial and imprecise probability for non-parametric modelling of rainfall in Manizales (Colombia). Ingeniería e Investigación, 28(2):22-29.

[5] Gabet, E. J., Burbank, D. W., Putkonen, J. K., Pratt-Sitaula, B. A. and Ojha, T. (2004). Rainfall thresholds for landsliding in the Himalayas of Nepal. Geomorphology, 63(3):131-143. DOI: 10.1016/j. geomorph.2004.03.011.

[6] Glade, T. (1998). Establishing the frequency and magnitude of landslidetriggering rainstorm events in New Zealand. Environmental Geology, 35(2-3):160-174. DOI: 10.1007/ s002540050302.

[7] Mateos, R. M., García-Moreno, I. and Azañón, J. M. (2012). Freeze-thaw cycles and rainfall as triggering factors of mass movements in a warm Mediterranean region: the case of the Tramuntana Range (Majorca, Spain). Landslides, 9(3):417432. DOI: 10.1007/s10346-011-0290-8.

[8] Pasuto, A. and Silvano, S. (1998). Rainfall as a trigger of shallow mass movements. A case study in the Dolomites, Italy. 
Environmental Geology, 35(2-3):184189. DOI: $10.1007 / \mathrm{s} 002540050304$.

[9] Terlien, M. T. J. (1996). Modelling Spatial and Temporal Variations in Rainfalltriggered Landslides: The Integration of Hydrologic Models, Slope Stability Models and Geographic Information Systems for the Hazard Zonation of Rainfall-triggered Landslides with Examples from Manizales (Colombia). International Institute for Aerial Survey and Earth Sciences (ITC).

[10] Caine, N. (1980). The rainfall intensity: duration control of shallow landslides and debris flows. Geografiska Annaler. Series A. Physical Geography, pp. 23-27. DOI: $10.2307 / 520449$.

[11] Glade, T., Crozier, M. and Smith, P. (2000). Applying probability determination to refine landslide-triggering rainfall thresholds using an empirical "antecedent daily rainfall model". Pure and Applied Geophysics, 157(6-8):1059-1079. DOl: $10.1007 / \mathrm{s} 000240050017$.

[12] Jakob, M. and Weatherly, H. (2003). A hydroclimatic threshold for landslide initiation on the North Shore Mountains of Vancouver, British Columbia. Geomorphology, 54(3):137-156. DOI: 10.1016/S0169-555X(02)00339-2.

[13] Aleotti, P. (2004). A warning system for rainfall-induced shallow failures. Engineering Geology, 73(3):247-265. DOI: 10.1016/j.enggeo.2004.01.007.

[14] Guzzetti, F., Peruccacci, S., Rossi, M. and Stark, C. P. (2008). The rainfall intensity - duration control of shallow landslides and debris flows: an update. Landslides, 5(1):317. DOI: 10.1007/s10346-007-0112-1.

[15] Brunetti, M., Peruccacci, S., Rossi, M., Luciani, S., Valigi, D. and Guzzetti, F. (2010). Rainfall thresholds for the possible occurrence of landslides in Italy. Natural Hazards and Earth System Science, 10(3):447-458.

[16] Li, C., Ma, T., Zhu, X. and Li, W. (2011). The power-law relationship between landslide occurrence and rainfall level. Geomorphology, 130(3):221-229. DOI: 10.1016/j.geomorph.2011.03.018.

[17] Mathew, J., Babu, D. G., Kundu, S., Kumar, K. V. and Pant, C. (2013). Integrating intensity-duration-based rainfall threshold and antecedent rainfall-based probability estimate towards generating early warning for rainfall-induced landslides in parts of the Garhwal Himalaya, India. Landslides, pp. 1-14. DOI: 10.1007/s10346-013-0408-2.

[18] Peel, M. C., Finlayson, B. L. and McMahon, T. A. (2007). Updated world map of the Köppen-Geiger climate classification. Hydrology \& Earth System Sciences Discussions, 4(2). DOl: 10.5194/hess11-1633-2007, 2007.

[19] Arango Gartner, J. D. et al. (2000). Relaciones lluvias-deslizamientos y zonificación geotécnica en la comuna dos de la ciudad de Manizales. Tesis de Maestría, Universidad Nacional de Colombia, sede Manizales.

[20] Bell, F. and Maud, R. (2000). Landslides associated with the colluvial soils 
overlying the Natal Group in the greater Durban region of Natal, South Africa. Environmental Geology, 39(9):10291038. DOI: $10.1007 / \mathrm{s002549900077.}$

[21] Govi, M. and Sorzana, P. (1980). Landslide susceptibility as a function of critical rainfall amount in piedmont basin (NorthWestern Italy). Studia Geomorphologica Carpatho-Balcanica, 14:43-61.

[22] Aristizábal, E., González, T., Montoya, J. D., Vélez, J. I., Martínez, H. and Guerra, A. (2011). Analysis of empirical rainfall thresholds for the prognosis of landslides in the Aburrá Valley, Colombia. Revista EIA, (15):95-111.

[23] $\mathrm{Au}$, S. (1998). Rain-induced slope instability in Hong Kong. Engineering Geology, 51(1):1-36. DOI: 10.1016/ 50013-7952(98)00038-6.

[24] Corominas, J. and Moya, J. (1999). Reconstructing recent landslide activity in relation to rainfall in the Llobregat river basin, Eastern Pyrenees, Spain. Geomorphology, 30(1):79-93. DOI: 10.1016/S0169-555X(99)00046-X.

[25] Díaz, E., De Olazagoitia, A. S., Ormaetxea, O. and Ibisate, A. (2012). Análisis de factores de desestabilización de laderas en dos cuencas del ámbito atlántico: Sollube-mape (Bizkaia) y Ramaio (Alava). Cuaternario y Geomorfología, 26(1):171-190.

[26] Echeverri, O. y Valencia, Y. (2004). Análisis de los deslizamientos en la cuenca de la quebrada La Iguaná de la ciudad de Medellín a partir de la integración lluviapendiente-formación geológica. Dyna, 71(142):33-45.
[27] Kirschbaum, D., Adler, R., Adler, D., Peters-Lidard, C. and Huffman, G. (2012). Global distribution of extreme precipitation and high-impact landslides in 2010 relative to previous years. Journal of Hydrometeorology, 13(5):15361551. DOl: http://dx.doi.org/10.1175/ JHM-D-12-02.1.

[28] Moreno, H. A., Vélez, M. V., Montoya, J. D. and Rhenals, R. L. (2006). La lluvia y los deslizamientos de tierra en Antioquia: análisis de su ocurrencia en las escalas interanual, intraanual y diaria. Revista EIA, 5:59-69.

[29] Sengupta, A., Gupta, S. and Anbarasu, K. (2010). Rainfall thresholds for the initiation of landslide at Lanta Khola in north Sikkim, India. Natural Hazards, 52(1):31-42. DOI: 10.1007/s11069009-9352-9.

[30] Cannon, S. H., Boldt, E. M., Laber, J. L., Kean, J. W. and Staley, D. M. (2011). Rainfall intensity-duration thresholds for postfire debris-flow emergencyresponse planning. Natural Hazards, 59(1):209-236. DOI: 10.1007/s11069011-9747-2.

[31] Lee, M. L., Ng, K. Y., Huang, Y. F. and Li, W. C. (2014). Rainfall-induced landslides in Hulu Kelang area, Malaysia. Natural Hazards, 70:1-23. DOI 10.1007/s11069013-0814-8.

[32] Crozier, M. and Eyles, R. (1980). Assessing theprobability of rapid mass movement.In Third Australia-New Zealand conference on Geomechanics: Wellington, May 1216, 1980, p. 2. Institution of Professional Engineers New Zealand. 
[33] Jaiswal, P. and van Westen, C. J. (2009). Estimating temporal probability for landslide initiation along transportation routes based on rainfall thresholds. Geomorphology, 112(1):96-105. DOl: 10.1016/j.geomorph.2009.05.008.

[34] Giannecchini, R. (2005). Rainfall triggering soil slips in the southern Apuan Alps (Tuscany, Italy). Advances in Geosciences, 2:21-24. DOI: 10.5194/ nhess-12-829-2012.

[35] Annunziati, A., Focardi, A., Focardi, P., Martello, S. and Vannocci, P. (2000). Analysis of the rainfall thresholds that induced debris flows in the area of Apuan Alps-Tuscany, Italy (19 June 1996 storm). In Proc. EGS Plinius Conf. on Mediterranean Storms, Maratea, Italy, pp. 485-493.

[36] Kanji, M., Massad, F. and Cruz, P. (2003). Debris flows in areas of residual soils: occurrence and characteristics. In International Workshop on Occurrence and Mechanism of Flows in Natural Slopes and Earthfills, pp. 1-13.

[37] Guidicini, G. and Iwasa, O. (1977). Tentative correlation between rainfall and landslides in a humid tropical environment. Bulletin of the International Association of Engineering GeologyBulletin de l'Association Internationale de Géologie de l'Ingénieur, 16(1):13-20. DOI: 10.1007/BF02591434.

[38] Zhou, W. and Tang, C. (2013). Rainfall thresholds for debris flow initiation in the Wenchuan earthquake-stricken area, southwestern China. Landslides, pp. 1-11. DOI: 10.1007/s10346-013-0421-5.
[39] Bacchini, M., Zannoni, A. et al. (2003). Relations between rainfall and triggering of debris-flow: case study of Cancia (Dolomites, Northeastern Italy). Natural Hazards and Earth System Science, 3(1/2):71-79.

[40] Akcali, E., Arman, H., Firat, S., Saltabas, L. and Gunduz, Z. (2010). Rainfall threshold for the initiation of landslides in Trabzon province of Turkey. International Journal of Engineering and Applied Science (IJEAC), 2(4):14-26.

[41] Ahmad, R. (2003). Developing early warning systems in Jamaica: rainfall thresholds for hydrological hazards. National Disaster Management Conference, Ocho Rios. St Ann, Jamaica, pp. 9-10.

[42] Baum, R. L. and Godt, J. W. (2010). Early warning of rainfall-induced shallow landslides and debris flows in the USA. Landslides, 7(3):259-272. DOI: 10.1007/ s10346-009-0177-0.

[43] Chien-Yuan, C., Tien-Chien, C., FanChieh, Y., Wen-Hui, Y. and Chun-Chieh, T. (2005). Rainfall duration and debris-flow initiated studies for real-time monitoring. Environmental Geology, 47(5):715-724. DOI: 10.1007/s00254-004-1203-0.

[44] Cannon, S. H. and Gartner, J. E. (2005). Wildfire-related debris flow from a hazards perspective. In Debris-flow Hazards and Related Phenomena, pp. 363-385. Springer.

[45] Cannon, S. H., Gartner, J. E., Wilson, R. C., Bowers, J. C. and Laber, J. L. (2008). Storm rainfall conditions for floods 
and debris flows from recently burned areas in southwestern Colorado and southern California. Geomorphology, 96(3):250-269. DOI: 10.1016/j. geomorph.2007.03.019.

[46] Coe, J. A., Kinner, D. A. and Godt, J. W. (2008). Initiation conditions for debris flows generated by runoff at Chalk Cliffs, central Colorado. Geomorphology, 96(3):270-297. DOI: 10.1016/j.geomor ph.2007.03.017.

[47] Dahal, R. K. and Hasegawa, S. (2008). Representative rainfall thresholds for landslides in the Nepal Himalaya. Geomorphology, 100(3):429-443. DOI: 10.1016/j.geomorph.2008.01.014.

[48] Giannecchini, R. (2006). Relationship between rainfall and shallow landslides in the southern Apuan Alps (Italy). Natural Hazards and Earth System Science, 6(3):357-364. DOI: 10.5194/ nhess-6-357-2006.

[49] Godt, J. W., Baum, R. L. and Chleborad, A. F. (2006). Rainfall characteristics for shallow landsliding in Seattle, Washington, USA. Earth Surface Processes and Landforms, 31(1):97-110. DOI: 10.1002/esp.1237.

[50] Hong, Y., Adler, R., and Huffman, G. (2006). Evaluation of the potential of NASA multisatellite precipitation analysis in global landslidehazardassessment. Geophysical Research Letters, 33(22):L22402. DOI: 10.1029/2006GL028010.

[51] Hong, Y., Hiura, H., Shino, K., Sassa, K., Suemine, A., Fukuoka, H., and Wang, G. (2005). The influence of intense rainfall on the activity of large-scale crystalline schist landslides in Shikoku Island, Japan. Landslides, 2(2):97-105. DOI: 10.1007/ s10346-004-0043-z.

[52] Jan, C.-D. and Chen, C.-L. (2005). Debris flows caused by Typhoon Herb in Taiwan. In Debris-Flow Hazards and Related Phenomena, pp. 539-563. Springer. DOI: 10.1007/3-540-27129-5_21.

[53] Khan, Y. A., Lateh, H., Baten, M. A., and Kamil, A. A. (2012). Critical antecedent rainfall conditions for shallow landslides in Chittagong City of Bangladesh. Environmental Earth Sciences, 67(1):97106. DOI: 10.1007/s12665-011-1483-0.

[54] Larsen, M. C. and Simon, A. (1993). A rainfall intensity-duration threshold for landslides in a humid-tropical environment, Puerto Rico. Geografiska annaler. Series A. Physical Geography, pp. 13-23.

[55] Marchi, L., Arattano, M., and Deganutti, A. M. (2002). Ten years of debris-flow monitoring in the Moscardo Torrent (Italian Alps). Geomorphology, 46(1):1-17. DOI: 10.1016/S0169-555X(01)00162-3.

[56] Miller, S., Brewer, T., and Harris, N. (2009). Rainfall thresholding and susceptibility assessment of rainfall-induced landslides: application to landslide management in St Thomas, Jamaica. Bulletin of Engineering Geology and the Environment, 68(4):539-550. DOI: 10.1007/s10064-009-0232-z.

[57] Paronuzzi, P., Coccolo, A., and Garlatti, G. (1998). Eventi meteorici critici e debris flows nei bacini Montani del Friuli. La Acqua, Sezione I-Memorie, pp. 39-50. 
[58] Rosi, A., Segoni, S., Catani, F., and Casagli, N. (2012). Statistical and environmental analyses for the definition of a regional rainfall threshold system for landslide triggering in Tuscany (Italy). Journal of Geographical Sciences, 22(4):617-629. DOI: 10.1007/s11442-012-0951-0.

[59] Ruiz-Villanueva, V., Bodoque, J., DíezHerrero, A. and Calvo, C. (2011). Triggering threshold precipitation and soil hydrological characteristics of shallow landslides in granitic landscapes. Geomorphology, 133(3):178-189. DOI: 10.1016/j.geomorph.2011.05.018.

[60] Saito, H., Nakayama, D. and Matsuyama, H. (2010). Relationship between the initiation of a shallow landslide and rainfall intensity-duration thresholds in Japan. Geomorphology, 118(1):167-175. DOI: 10.1016/j.geomorph.2009.12.016.

[61] Tiranti, D. and Rabuffetti, D. (2010). Estimation of rainfallthresholdstriggering shallow landslides for an operational warning system implementation. Landslides, 7(4):471-481. DOI: 10.1007/ s10346-010-0198-8.

[62] Zezere, J., Trigo, R., Trigo, I. et al. (2005). Shallow and deep landslides induced by rainfall in the Lisbon region (Portugal): assessment of relationships with the North Atlantic Oscillation. Natural Hazards and Earth System Science, 5(3):331-344. DOI: 10.5194/nhess-5-331-2005.

[63] Dai, F. and Lee, C. (2001). Frequencyvolume relation and prediction of rainfallinduced landslides. Engineering Geology, 59(3):253-266. DOI: 10.1016/S00137952(00)00077-6.

[64] Ni, H., Zheng, W., Li, Z. and Ba, R. (2010). Recent catastrophic debris flows in Luding County, SW China: geological hazards, rainfall analysis and dynamic characteristics. Natural Hazards, 55(2):523-542. DOI: 10.1007/s11069010-9545-2.

[65] Sepúlveda, S. A. and Padilla, C. (2008). Rain-induced debris and mudflow triggering factors assessment in the Santiago cordilleran foothills, Central Chile. Natural Hazards, 47(2):201-215. DOI: 10.1007/s11069-007-9210-6.

[66] García, H. A., Colonia, J. D. and Corrales, J. J. (2013). Plataforma tecnológica para el monitoreo de amenazas naturales en el oleoducto Ocensa. In UPTC, editor, Memorias del 3er. Seminario internacional de ingeniería sísmica y geotecnia, 1-12, Tunja, Colombia. 